\title{
HOMOTOPY GROUPS OF CERTAIN DELETED PRODUCT SPACES
}

\author{
C. W. PATTY ${ }^{1}$
}

If $X$ is a topological space, let $D_{X}$ denote the subset of $X \times X$ consisting of the set of all points of the form $(x, x)$, where $x \in X$. Then the deleted product space, $X^{*}$, of $X$ is the space $X \times X-D_{X}$ with the relative topology. It follows from a theorem of Eilenberg (see [1, p. 43]) that for a connected, finite, 1-dimensional polyhedron $X, X^{*}$ is arcwise connected if and only if $X$ is not an arc.

In this paper we prove the following theorem:

If $X$ is a connected, finite, 1-dimensional polyhedron which is not an arc, then $\Pi_{k}\left(X^{*}\right)=0$ for all $k>1$.

Definition 1. If $X$ is a connected, finite, 1-dimensional polyhedron and $A$ and $B$ are subpolyhedra of $X$, let $P\left(A \times B-D_{X}\right)$ $=U\{r \times s \mid r$ is a simplex of $A, s$ is a simplex of $B$, and $r \cap s=\varnothing\}$.

REMARK 1. Let $X$ be a connected, finite, 1-dimensional polyhedron which is not an arc. If $X$ does not have a vertex of order $\geqq 3$, then $X$ is a simple closed curve. If $X$ does have a vertex of order $\geqq 3$, let $A^{\prime}$ be a triod in $X$. Then it is clear that there is a subdivision $X^{\prime}$ of $X$ such that: (1) each simplex of $A^{\prime}$ is a simplex of $X^{\prime}$, (2) $X^{\prime}$ consists of a finite number of 1 -simplexes, $r_{1}, \cdots, r_{k}$, and (3) $X^{\prime}$ can be realized by starting with $A^{\prime}$ and adding one 1 -simplex $r_{j}$ at a time so that either $r_{j} \cap\left(\bigcup_{k=1}^{j-1} r_{k}\right)$ is a single vertex or $r_{j} \cap\left(\bigcup_{k=1}^{j-1} r_{k}\right)$ consists of two vertices $v_{1}, v_{2}$, where each $v_{i}(i=1,2)$ is a vertex of order one in $\bigcup_{k=1}^{j-1} r_{k}$. In this paper, we shall assume that such a subdivision of $X$ has been made.

Definition 1 and Remark 1 may be found in [3]. It is shown in [4] that if $X$ is a connected, finite, 1-dimensional polyhedron, then there is a deformation retraction of $X^{*}$ onto $P\left(X^{*}\right)$.

Definition 2. A space $X$ is said to be aspheric if $\Pi_{k}(X)=0$ for all $k>1$.

Theorem 1. Let $X=A \cup B$, where $X, A$, and $B$ are connected polyhedra and $A \cap B$ has a finite number of components $C_{i}$. Suppose that

(1) $A, B$, and all the $C_{i}$ are aspheric.

(2) For each of the $C_{i}$, the injections

Received by the editors July 29, 1960. tion.

1 This work was partially supported by the University of Georgia Alumni Founda- 


$$
\begin{aligned}
& i_{*}^{1}: \mathrm{I}_{1}\left(C_{i}\right) \rightarrow \mathrm{I}_{1}(A) \text { and } \\
& i_{*}^{2}: \mathrm{II}_{1}\left(C_{i}\right) \rightarrow \mathrm{I}_{1}(B) \text { are isomorphisms into. }
\end{aligned}
$$

Then $X$ is aspheric.

The above theorem is due to J. H. C. Whitehead [5, p. 159].

Theorem 2. If $X$ is a connected, finite, 1-dimensional polyhedron which is not an arc, then $X^{*}$ is aspheric.

Proof. The author [3] has shown that if $X$ is either a simple closed curve or a triod, then $X^{*}$ has the homotopy type of a simple closed curve.

The proof is by induction on the number of 1 -simplexes of $X$. Suppose that the theorem is true if $X$ consists of $n-11$-simplexes, where $n \geqq 4$. Now suppose $X$ is not a simple closed curve and $X$ consists of $n$ 1-simplexes. By Remark 1 , it is possible to express $X$ as $X=A \cup B$, where $A$ is a connected polyhedron which is the union of $n-1$ 1simplexes, $B$ is a 1 -simplex, and either (1) $A \cap B=\{v\}$, where $v$ is a vertex of $X$, or (2) $A \cap B=\left\{v_{1}\right\} \cup\left\{v_{2}\right\}$, where $v_{1}$ and $v_{2}$ are vertices of order 2 in $X$. It is possible to choose $A$ in such a manner that $A$ contains a vertex of order $\geqq 3$, and we assume that this has been done.

CASE (1). It is easy to see that

$$
P\left(X^{*}\right)=P\left(A^{*}\right) \cup P\left(B \times A-D_{X}\right) \cup P\left(A \times B-D_{X}\right)
$$

and

$$
P\left(A^{*}\right) \cap P\left(B \times A-D_{X}\right)=P\left(v \times A-D_{X}\right) .
$$

First we show that

$$
P\left(A^{*}\right) \cup P\left(B \times A-D_{X}\right)=P\left(X \times A-D_{X}\right)
$$

is aspheric. Let $u$ be the other vertex of $B$. Then $u \times A C_{P}\left(B \times A-D_{X}\right)$, and it is easy to see that there is a deformation retraction of $P\left(B \times A-D_{X}\right)$ onto $u \times A$. Therefore $P\left(B \times A-D_{X}\right)$ and $u \times A$ have the same homotopy type. Hence $\Pi_{k}\left(P\left(B \times A-D_{X}\right)\right)=0$ for all $k>1$ since $u \times A$ is a 1 -dimensional polyhedron.

Let $C_{1}, \cdots, C_{q}$ denote the components of $P\left(v \times A-D_{\mathbf{X}}\right)$. Each $C_{i}$ is aspheric since it is a 1-dimensional polyhedron. For each $C_{i}$, consider the diagram

$$
\Pi_{1}\left(C_{i}\right) \stackrel{j_{*}^{1 i}}{\rightarrow} \Pi_{1}(v \times A) \stackrel{j_{*}^{2}}{\rightarrow} \Pi_{1}(A \times A)
$$

where $j_{*}^{1 t}$ and $j_{*}^{2}$ are the injection homomorphisms. It is clear that $j_{*}^{1 i}$ is an isomorphism into since $C_{i}=v \times A_{i}$, where $A_{i}$ is a subpolyhedron 
of $A$, and it is a well-known result that $j_{*}^{2}$ is an isomorphism into. Therefore $j_{*}^{2} j_{*}^{1 i}$ is an isomorphism into.

Now consider the diagram

$$
\mathrm{II}_{1}\left(C_{i}\right) \stackrel{k_{*}^{1 i}}{\rightarrow} \mathrm{II}_{1}\left(P\left(A^{*}\right)\right) \stackrel{j^{3}}{\rightarrow} \mathrm{II}_{1}(A \times A)
$$

where $k_{*}^{1 i}$ and $j_{*}^{3}$ are injection homomorphisms. By the above argument $k_{*}^{1 i} j_{*}^{3}$ is an isomorphism into. Hence $k_{*}^{1 i}$ is an isomorphism into.

Now consider the diagrams

$$
\begin{gathered}
\Pi_{1}\left(C_{i}\right) \rightarrow \Pi_{1}(v \times A) \rightarrow \Pi_{1}(B \times A), \\
\Pi_{1}\left(C_{i}\right) \stackrel{k_{*}^{2 i}}{\rightarrow} \Pi_{1}\left(P\left(B \times A-D_{X}\right)\right) \rightarrow \Pi_{1}(B \times A)
\end{gathered}
$$

where each indicated homomorphism is the injection homomorphism. By essentially repeating the above argument with respect to the new diagrams, it can be shown that $k_{*}^{2 t}$ is an isomorphism into.

Therefore the conditions of Theorem 1 are satisfied, and hence $P\left(X \times A-D_{X}\right)$ is aspheric.

Now we consider

$$
P\left(X \times A-D_{X}\right) \cup P\left(A \times B-D_{X}\right)=P\left(X^{*}\right) .
$$

Observe that

$$
P\left(X \times A-D_{X}\right) \cap P\left(A \times B-D_{X}\right)=P\left(A \times v-D_{X}\right) .
$$

Let $D_{1}, \cdots, D_{q}$ denote the components of $P\left(A \times v-D_{X}\right)$. Again $P\left(A \times B-D_{X}\right)$ and $D_{i}(i=1, \cdots, q)$ are aspheric. In order to show that $P\left(X^{*}\right)$ is aspheric, we consider the four diagrams

$$
\begin{gathered}
\Pi_{1}\left(D_{i}\right) \rightarrow \Pi_{1}(A \times v) \rightarrow \Pi_{1}(X \times A), \\
\Pi_{1}\left(D_{i}\right) \stackrel{m_{*}^{1 i}}{\longrightarrow} \Pi_{1}\left(P\left(X \times A-D_{X}\right)\right) \longrightarrow \Pi_{1}(X \times A), \\
\Pi_{1}\left(D_{i}\right) \rightarrow \Pi_{1}(A \times v) \rightarrow \Pi_{1}(A \times B), \\
\Pi_{1}\left(D_{i}\right) \stackrel{m_{*}^{2 i}}{\longrightarrow} \Pi_{1}\left(P\left(A \times B-D_{X}\right)\right) \longrightarrow \Pi_{1}(A \times B)
\end{gathered}
$$

where each indicated homomorphism is the injection homomorphism.

Using these four diagrams, we essentially repeat the above argument to show that $m_{*}^{1 i}$ and $m_{*}^{2 i}$ are isomorphisms into. Then, by Theorem $1, P\left(X^{*}\right)$ is aspheric.

C.ASE (2). It is easy to see that 


$$
P\left(X^{*}\right)=P\left(A^{*}\right) \cup P\left(B \times A-D_{X}\right) \cup P\left(A \times B-D_{X}\right)
$$

and

$$
P\left(A^{*}\right) \cap P\left(B \times A-D_{X}\right)=P\left(v_{1} \times A-D_{X}\right) \cup P\left(v_{2} \times A-D_{X}\right) .
$$

First we show that

$$
P\left(A^{*}\right) \cup P\left(B \times A-D_{X}\right)=P\left(X \times A-D_{X}\right)
$$

is aspheric. For $i=1,2, P\left(v_{i} \times A-D_{X}\right)$ is a connected, 1-dimensional polyhedron, and hence it is aspheric. Let $A^{\prime}$ be the subpolyhedron of $A$ consisting of the union of all the 1-simplexes in $A$ except those two which have $v_{1}$ and $v_{2}$ as vertices. Then $v_{1} \times A^{\prime} \subset P\left(B \times A-D_{X}\right)$, and it is easy to see that there is a deformation retraction of $P\left(B \times A-D_{X}\right)$ onto $v_{1} \times A^{\prime}$. Hence $\Pi_{k}\left(P\left(B \times A-D_{X}\right)\right)=0$ for all $k>1$ since $v_{1} \times A^{\prime}$ is a connected, 1-dimensional polyhedron.

Consider the diagram

$$
\Pi_{1}\left(P\left(v_{i} \times A-D_{X}\right)\right) \stackrel{k_{*}^{1 i}}{\rightarrow} \Pi_{1}\left(P\left(A^{*}\right)\right) \stackrel{j_{*}^{1}}{\rightarrow} \Pi_{1}(A \times A)
$$

where $k_{*}^{1 i}$ and $j_{*}^{1}$ are the injection homomorphisms. Now $j_{*}^{1} k_{*}^{1 t}$ is an isomorphism into since $P\left(v_{i} \times A-D_{X}\right)$ and $v_{i} \times A$ have the same homotopy type. Therefore $k_{*}^{1 i}$ is an isomorphism into. By considering a similar diagram, it is easy to see that the injection homomorphism

$$
k_{*}^{2 i}: \Pi_{1}\left(P\left(v_{i} \times A-D_{X}\right)\right) \rightarrow \Pi_{1}\left(P\left(B \times A-D_{X}\right)\right)
$$

is an isomorphism into. Hence the conditions of Theorem 1 are satisfied, and therefore $P\left(X \times A-D_{X}\right)$ is aspheric.

Now we consider

$$
P\left(X \times A-D_{\mathbf{X}}\right) \cup P\left(A \times B-D_{\mathbf{X}}\right)=P\left(X^{*}\right) .
$$

By essentially repeating the above argument, it can be shown that $P\left(X^{*}\right)$ is aspheric.

Example. Let $X$ be the polyhedron shown in the following diagram:

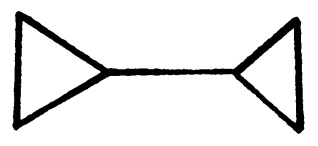

Then $H_{2}\left(X^{*}, Z\right)$ is a free abelian group of rank 2 [2, p. 364]. Therefore $X^{*}$ does not admit a 1 -complex as deformation retract. This answers in the negative a question asked by the referee. 


\section{BIBLIOGRAPHY}

1. S. Eilenberg, Ordered topological spaces, Amer. J. Math. vol. 63 (1941) pp. 3945.

2. S. T. Hu, Isotopy invariants of topological spaces, Proc. Royal Soc. London Ser. A vol. 255 (1960) pp. 331-366.

3. C. W. Patty, The fundamental group of certain deleted product spaces, to appear.

4. A. Shapiro, Obstructions to the imbedding of a complex in a euclidean space: I. The first obstruction, Ann. of Math. vol. 66 (1957) pp. 256-269.

5. J. H. C. Whitehead, On the asphericity of regions in a 3-sphere, Fund. Math. vol. 32 (1939) pp. 149-166.

The University of Georgia and

The University of North Carolina 